\title{
Particle Removal by Electrostatic and Dielectrophoretic Forces for Dust Control During Lunar Exploration Missions
}

\author{
C.I. Calle ${ }^{a}$ C.R. Buhler ${ }^{b}$, J.L. McFall ${ }^{b}$, and S.J. Snyder \\ ${ }^{a}$ NASA Electrostatics and Surface Physics Laboratory, Kennedy Space Center, FL 32899 \\ ${ }^{\mathrm{b}}$ ASRC Aerospace, Kennedy Space Center, FL 32899
}

\begin{abstract}
Particle removal during lunar exploration activities is of prime importance for the success of robotic and human exploration of the moon. We report on our efforts to use electrostatic and dielectrophoretic forces to develop a dust removal technology that prevents the accumulation of dust on solar panels and removes dust adhering to those surfaces. Testing of several prototypes showed solar shield output above $\mathbf{9 0 \%}$ of the initial potentials after dust clearing.
\end{abstract}

Keywords: Dielectrophoretic forces, Electric fields, Particle charging, Space Applications

\section{Introduction}

Since the moon has an extremely rarefied atmosphere, the full spectrum of the sun's electromagnetic radiation reaches the surface, charging the surface dust and affecting its current charge state. It is thus very likely that the lunar surface dust remains electrostatically charged at all times. Whether charged or uncharged, lunar dust will present several challenges to manned and unmanned exploration missions currently being planned. Dust will adversely affect the operation of most mechanical systems required by these missions. Charged dust will be particularly difficult to remove from astronauts suits, gloves, and visors. Charged dust will also stubbornly adhere to solar panels and thermal radiators, thus decreasing their efficiencies. 
In an effort to mitigate the dust problem, the electrodynamic dust shield, an active dust mitigation technology, is currently being developed in our laboratory. This technology is proving to be very effective in the removal of dust particles from surfaces and in the prevention of the accumulation of those particles on such surfaces. The technology makes use of electrostatic and dielectrophoretic forces to move charged dust particles off surfaces and to prevent dust particles from depositing on those surfaces.

\section{Background and Theory}

The electrodynamic dust shield technology described in this paper is based on the electric curtain concept developed by F.B. Tatom and collaborators at NASA in 1967 [1] and further developed by Masuda at the University of Tokyo in the 1970s [2-6]. This technique has been shown to lift and transport charged and uncharged particles using electrostatic and dielectrophoretic forces $[7,8]$. The technology has never been applied to space applications on the moon.

The electric curtain consists of a series of parallel electrodes connected to a multi-phase AC source. This source generates a traveling wave that carries dust particles along (Fig. 1). In general, the net force of repulsion on the particles, which levitates them above the surface, can be expressed as the contribution from the electrodynamic force, the viscous force, and the gravitational force:

$$
M \frac{d^{2} r}{d t^{2}}=q E \cos \omega t-6 \pi \eta \frac{d r}{d t}-m g
$$

where $m$ is the particle mass, $r$ is the particle's position, $\eta$ is the viscosity of the fluid in which the particles move, $q$ is the particle charge, and $g$ is the acceleration due to gravity. In the rarefied lunar atmosphere, the viscous force is negligible and this equation becomes:

$$
M \frac{d^{2} r}{d t^{2}}=q E \cos \omega t-m g
$$




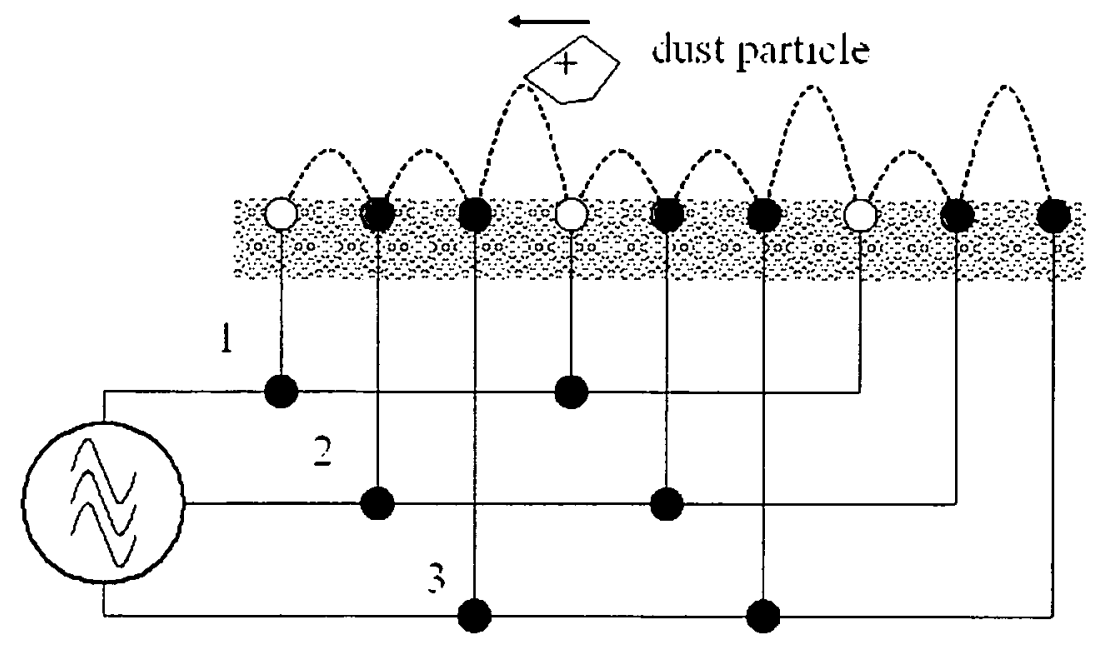

Fig. 1: Three-phase electric curtain.

Due to the complicated nature of the particle-field interaction, where the motion of the particles is nonlinear and coupled, this equation of motion cannot be solved analytically. Masuda [9] proposed a solution to a linear approximation to the equation of motion assuming small oscillations for the particles. With a numerical solution to the equation of motion, Masuda was able to obtain simulations of the particle motion which matched actual measurements of particle trajectories fairly well.

Although the forces responsible for the levitation of the particles are highly dependent on their charge, uncharged particles can ultimately be removed from the curtain as well. It has been well documented that polarizable particles can be levitated using these techniques [10]. Since many larger neutral particles contain nearly equal amounts of positive and negative charges on their surface, these particles possess an extrinsic electric dipole moment. If this dipole moment is exposed to a spatially non-uniform electric field, the particles will experience a force. Likewise, particles with intrinsic electric dipole moments or contaning polar materials like water will also experience a force. The movement of particles with internal electric dipole moments in a non-uniform electric field is called the dielectrophoretic force [10]. All that is required for levitation is that the particles have a different dielectric constant than that of the surrounding medium. The time-averaged force of an electric dipole in a spatially (and time) dependent electric field is given by

$$
\langle\bar{F}\rangle=\frac{1}{2} \operatorname{Re}\left[(\vec{p} \cdot \bar{\nabla}) \cdot \bar{E}^{*}\right]
$$


where $\vec{E}^{*}$ is the complex conjugate of the electric field and $\vec{p}$ is the induced electric dipole moment. For spherical particles the dipole moment becomes

$$
\vec{p}=4 \pi \varepsilon_{m} r^{3} f_{\mathrm{CM}} \vec{E}
$$

where $\varepsilon_{m}$ is the permittivity of the medium and $f_{\mathrm{CM}}$ is the Clausius-Mossotti factor given by:

$$
f_{\mathrm{CM}}=\frac{\varepsilon_{p}^{*}-\varepsilon_{m}^{*}}{\varepsilon_{p}^{*}+2 \varepsilon_{m}^{*}}
$$

Here $\varepsilon_{p}^{*}$ and $\varepsilon_{m}^{*}$ are the complex permittivities of the particle and the medium, respectively. Combining the above equations yields the following result for the time-averaged dielectrophoretic force experienced by polarizable spherical particles:

$$
\langle\vec{F}\rangle=\pi \varepsilon_{m} r^{3}\left[\operatorname{Re}\left(f_{\mathrm{CM}}\right) \nabla \vec{E}^{2}+2 \operatorname{Im}\left(f_{\mathrm{CM}}\right) \nabla \times\left(\vec{E}_{\mathrm{I}}^{\cdot} \times \vec{E}_{\mathrm{R}}\right)\right]
$$

$\vec{E}_{1}$ and $\vec{E}_{\mathrm{R}}$ are the negative gradients of the potentials $\phi_{1}$ and $\phi_{\mathrm{R}}$, while $\operatorname{Re}\left(f_{\mathrm{CM}}\right)$ and $\operatorname{Im}\left(f_{\mathrm{CM}}\right)$ are the real and imaginary parts of the Clausius-Mossotti factor, respectively [6]. This force not only applies to polarizable particles, but also to bipolar particles (those containing equal amounts of positive and negative charge) and this component should be added to the force equation. If the permittivities of the particles are less than that of the medium, the particles will move toward the point in which the field gradient is the smallest, i.e. away from the curtain. However, in a majority of cases, the particles have a higher dielectric constant than the surrounding medium and will be attracted to the curtain's electrodes. In this case, a neutral particle traveling along the insulated screen would triboelectrically acquire a charge and would then be lifted from the screen by the stronger $q E$ force. Solving the equation of motion containing just the dielectrophoretic force alone is extremely difficult analytically and requires computational methods.

The dielectrophoretic force is commonly used for transportation of particles in a liquid medium [8], fluids through microchannels [5], blood cells [11] and other biological matter [12]. 


\section{Experiments}

Electrodynamic dust shield prototypes based on the electric curtain concept have been developed in our laboratory. These prototypes can remove dust from surfaces and also prevent dust accumulation. Several types of dust shields were designed and built. Rigid, opaque shields in a dielectric substrate with metallic electrodes in parallel or spiral configurations; rigid, transparent shields on a dielectric substrate with transparent electrodes in a parallel or spiral configuration; rigid, transparent shields in a dielectric coating on a metallic substrate with metallic electrodes in a parallel or spiral configuration; flexible, opaque shields on a dielectric substrate with flexible electrodes; and flexible, transparent shields on a dielectric substrate with flexible, transparent electrodes.

We have tested these dust shield configurations under high vacuum conditions and under simulated lunar gravity to validate the technology for lunar exploration applications [13-18]. Other laboratories have developed similar techniques and have achieved some success under ambient conditions $[19,20]$. In this paper, we describe the implementation of our dust shields to solar panels and their performance under pressures of the order of $10^{-6} \mathrm{kPa}$.

Prevention of dust accumulation on solar panels for future lunar exploration missions constitutes one of the most important applications of our dust shield technology. To provide a proof of concept, $5 \mathrm{~cm} \times 8 \mathrm{~cm}$ dust shields were fabricated using transparent indium tin oxide (ITO) electrodes on glass substrates. These dust shields were placed on off-the-shelf $5 \mathrm{~cm} \times 5 \mathrm{~cm}$ solar panels capable of providing potentials of 3 to 4 volts. The shield electrodes had trace widths between 0.3 and $0.4 \mathrm{~mm}$ and spacing varying between 0.48 and 0.67 $\mathrm{mm}$. All dust shields were powered by a three-phase square-wave signal and laid out in the form of a spiral pattern to allow electrical contact to be made on one side of the surface (Fig. 2). The connectors were attached using silver paint for conductivity and coated with epoxy to prevent breakdown and to provide mechanical stability. 


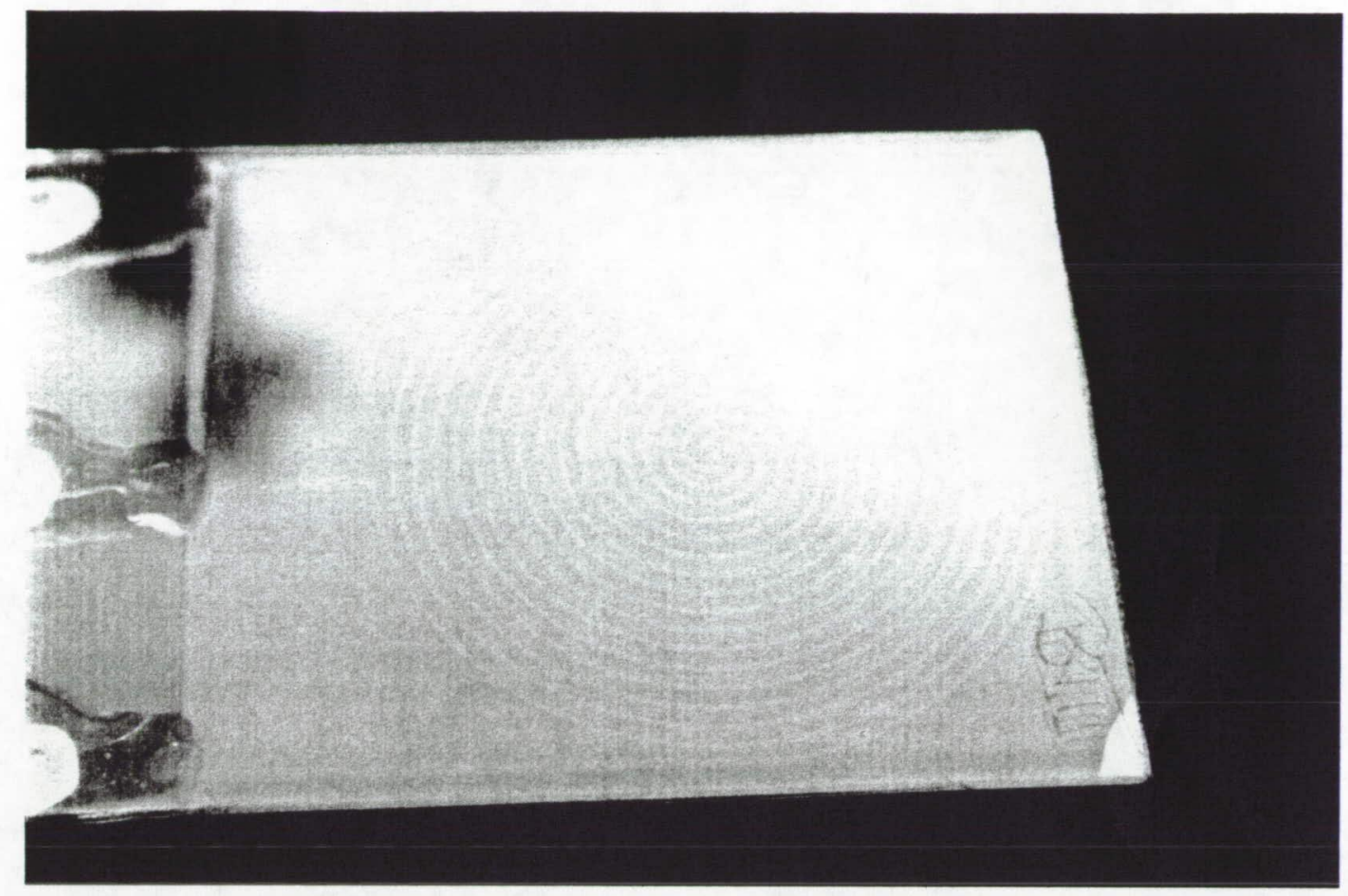

Fig. 2. Three-phase transparent dust shield with a spiral configuration of three ITO electrodes on glass.

For testing at high vacuum, commercial polyester laminate in two thicknesses, $1.3 \mu \mathrm{m}$ and $1.8 \mu \mathrm{m}$, was used to coat the shields. Laminate is an excellent choice of coating for testing purposes since it has a moderate dielectric breakdown strength, is easy to implement, and is relatively transparent to visible light. Testing was performed in a vacuum chamber at $10^{-6} \mathrm{kPa}$. JSC-1A lunar simulant - a granular material especially prepared with chemical characteristics that are close to those of the Apollo lunar samples-was kept in a vacuum oven for several days prior to each experiment [21].

Approximately $20 \mathrm{mg}$ of JSC-1A simulant dust in the $50 \mu \mathrm{m}-75 \mu \mathrm{m}$ size fraction were delivered to each dust shield by rapidly shaking a feeder cup $4 \mathrm{~cm}$ in diameter containing a metal screen mesh. The mesh sizes were chosen for their ability to contain dust before the shaker was activated. Shaking of the feeder cup occurred by engaging a vibrating motor fastened to the cup. Dust was deposited to the surface of each shield individually while under vacuum conditions using two computer-controlled translation stages $(X$ and $Y$ direction). The translation stages positioned the shaker feeder over the shield, deposited dust, and moved to 
the next shield. Once dust was deposited on all four shields, the stages were sent to the "home" position to allow for visual inspection and videography.

The demonstration of clearing efficiencies was performed by measuring the output of the solar panels. Aluminum foil cutouts were used to match the spiral pattern of the shields for efficiency measurements (Fig. $3)$.

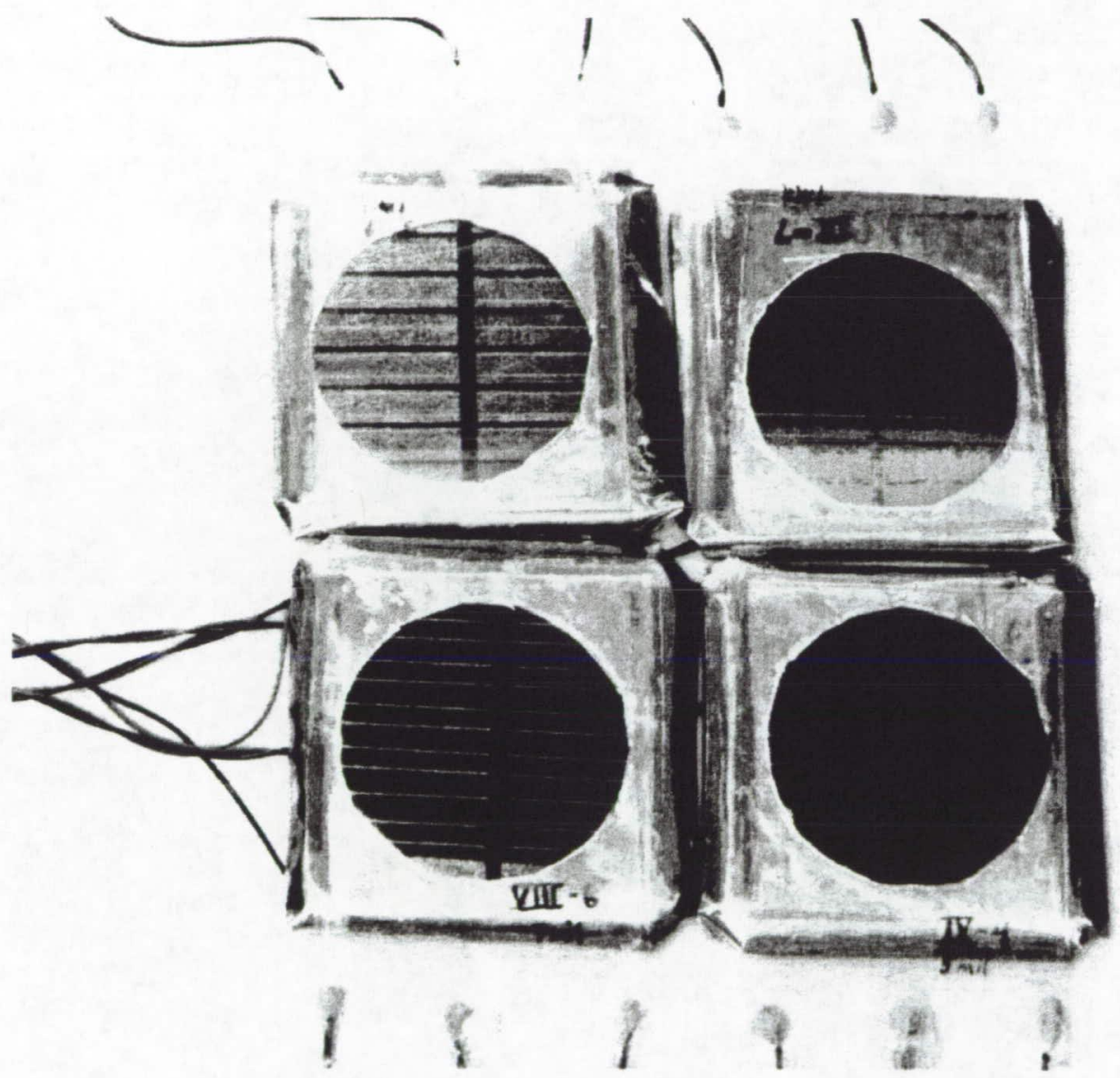

Fig. 3. Transparent dust shields on commercial solar panels used for testing at high vacuum conditions. Aluminum foil cutouts were placed on the dust shields to cover areas without electrodes.

Fig. 4 shows the solar panel response throughout the experiments. The time offset in voltage level drop for each panel is due to the time it took for the feeder cup to deliver dust to each shield individually. As shown in Fig. 4, the deposition of JSC-1A dust corresponds to a significant drop in output voltage for each solar panel. The voltage output typically drops to or below $20 \%$ of its initial voltage. Once the electrodynamic dust shields are turned on, the voltages raise quickly to above $90 \%$ of the initial values within the first two 
minutes. Fig. 5 shows the solar panel response for one of the four different electrode configurations. The baseline voltage of the solar panels without dust layering is shown for comparison. Measurements of the solar cell performance taken after dust loading and after dust shield activation are shown. Performance measurements are given relative to solar cell baseline performance.

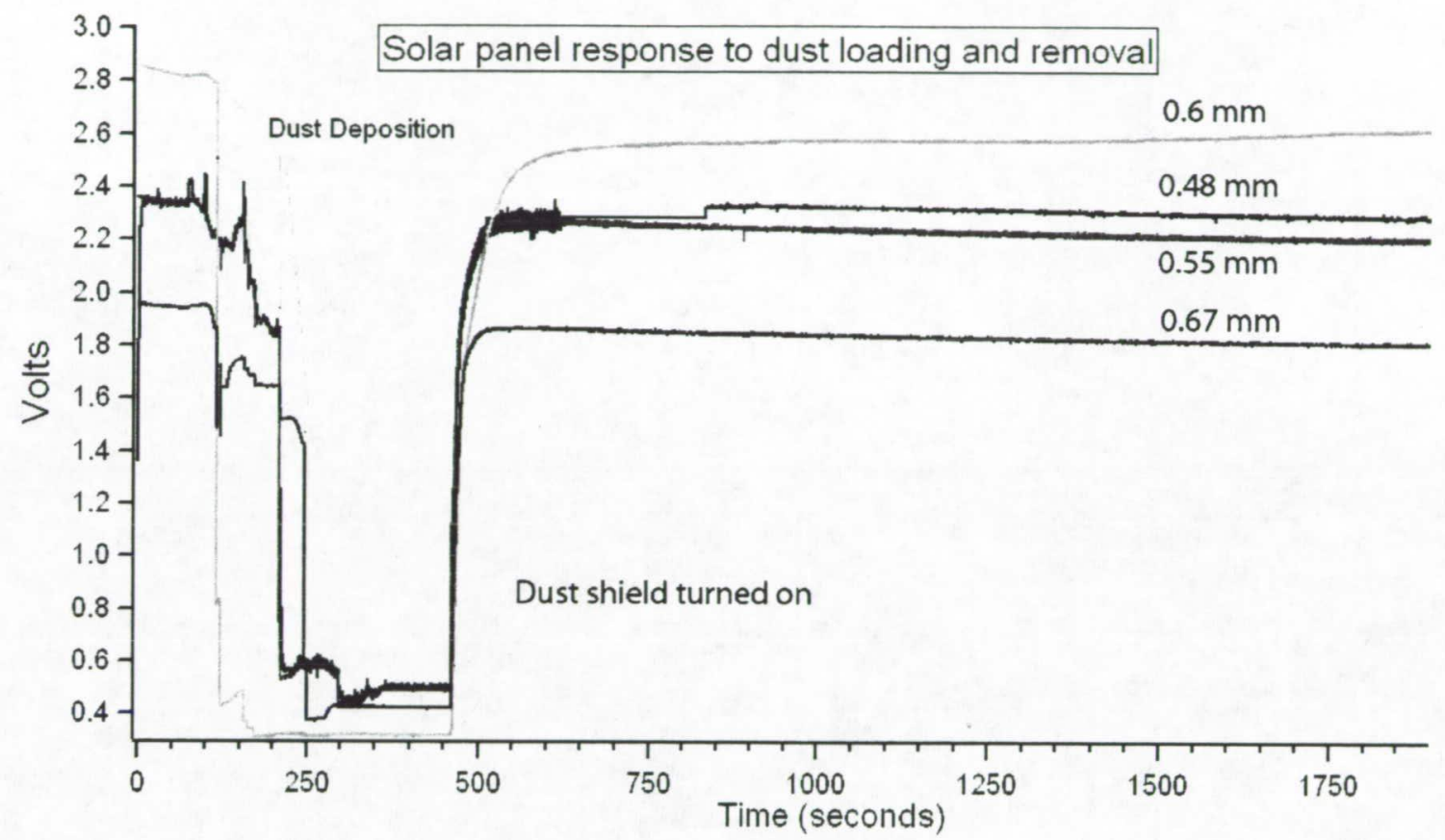

Fig. 4. Solar panel response to 50-75 $\mu \mathrm{m}$ JSC-1A dust deposition and removal under high vacuum conditions. Removal was accomplished using dust shields of four different electrode spacings: $0.48 \mathrm{~mm}, 0.55 \mathrm{~mm}, 0.6 \mathrm{~mm}$, and $0.67 \mathrm{~mm}$. 


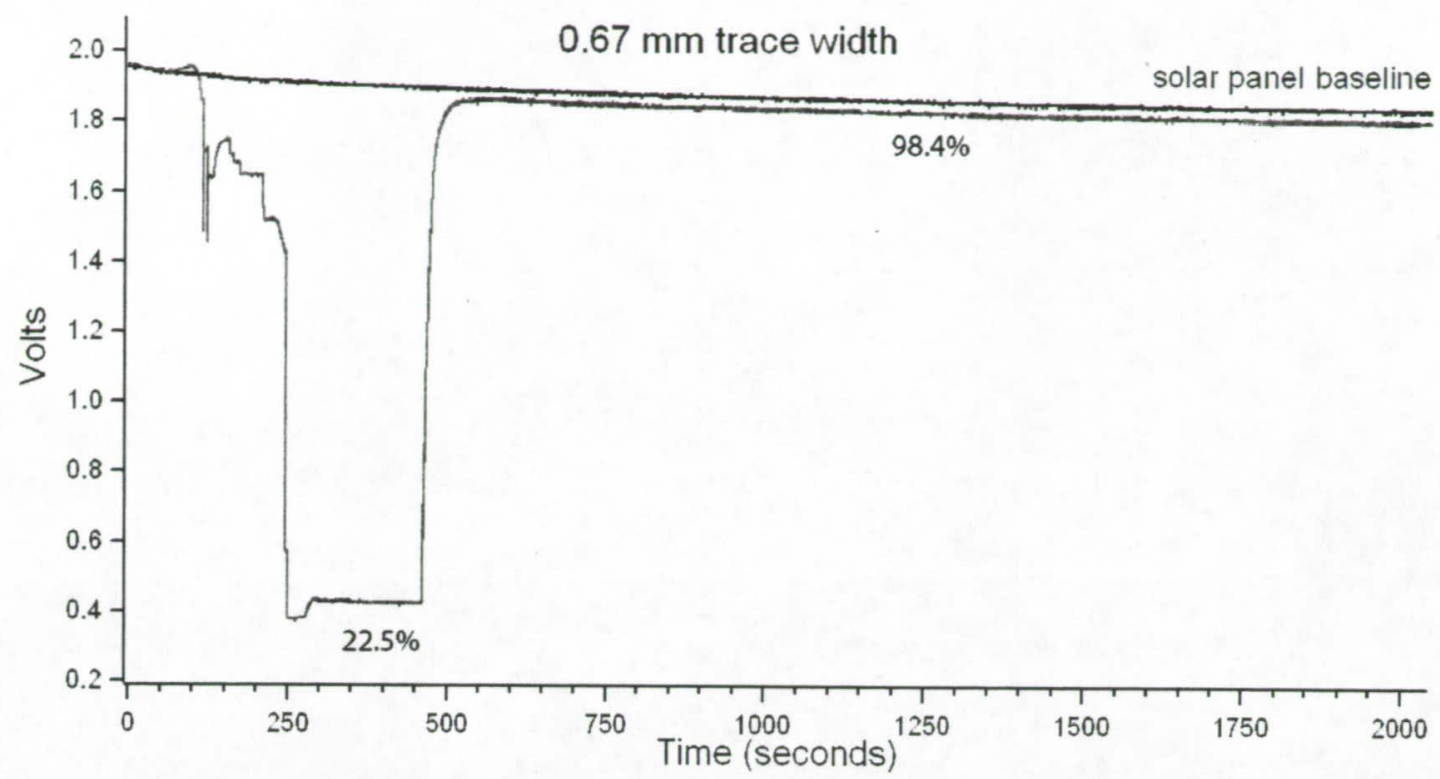

Fig. 5. Solar panel response to JSC-1A dust loading and removal with $0.67 \mathrm{~mm}$ trace. Baseline voltages are shown for comparison.. Performance is relative to baseline values.

Table 1 shows the reduced efficiencies as a function of dust loading for each of the four shields. Dust loading is typically quite dramatic, covering most of the area of the solar panel. After the shields are activated, the voltage recovers to above $90 \%$ of the initial voltage. The error in the data is on the order of $2 \%$.

Table 1. Solar panel performance.

\begin{tabular}{|c|c|c|}
\hline $\begin{array}{c}\text { Solar Panel Trace } \\
\text { Width }\end{array}$ & $\begin{array}{c}\text { Performance with } \\
\text { Dust Deposition }\end{array}$ & $\begin{array}{c}\text { Performance after Dust } \\
\text { Shield Activation }\end{array}$ \\
\hline $0.48 \mathrm{~mm}$ & $20.3 \%$ & $99.4 \%$ \\
\hline $0.55 \mathrm{~mm}$ & $19.3 \%$ & $98.7 \%$ \\
\hline $0.6 \mathrm{~mm}$ & $11.0 \%$ & $91.6 \%$ \\
\hline $0.67 \mathrm{~mm}$ & $22.5 \%$ & $98.4 \%$ \\
\hline
\end{tabular}

\section{Conclusions}

Lunar exploration missions may be hindered by the presence of highly charged lunar dust that will adhere electrostatically to the surfaces of equipment, viewports, optical systems, instrumentation, and spacesuits. In this paper, we have reported on an active dust removal and prevention system that we have been developing over several years. These dust shield systems use electrostatic and dielectrophoretic forces to remove dust 
already adhering to surfaces and to prevent the accumulation of dust approaching such surfaces. We describe the implementation of these dust shields to solar panels.

Our results show that the transparent dust shields applied to commercial solar panels operate successfully under high vacuum even under extreme dust loading conditions that caused the solar cell performance to drop to 11 to $23 \%$ of the baseline performance. After dust shield activation, the solar cell performance increased to values above $90 \%$. The application of the dust shields did not appear to have any adverse effects on the behavior of the solar panels. Further analysis will need to be performed to determine any electromagnetic interference or electromagnetic compatibility issues that may arise when the shield technology is incorporated into a full photovoltaic system. Optimization of the different techniques that we have developed is currently underway. Further development of the dust shields continues with applications to thermal radiators and other systems.

\section{References}

[1] Tatom, F.B., V. Srepel, R.D. Johnson, N.A. Contaxes, J.G. Adams, H. Seaman, and B.L. Cline, "Lunar Dust Degradation Effects and Removal/Prevention Concepts", NASA Technical Report No. TR-792-7-207A, p. 3-1 (1967).

[2] Masuda, S., Advances in Static Electricity, 1, Auxilia, S.A., Brussels, 398 (1970)

[3] Masuda, S. Fujibayashi, K., Ishida, K., and Inaba, H., Electronic Engineering in Japan, 92, 9 (1972).

[4] Masuda, S., and Matsumoto, Y., Proc .of the $2^{\text {nd }}$ International on Static Electrification, Frankfurt (1973)

[5] Masuda, S., Washizu, M., Kawabata, I., "Movement of Blood Cells in Liquid by Nonuniform Traveling Field”, IEEE Transactions on Industrial Applications, 24, No. 2, pp. 217-222 (1988)

[6] Aoyoma, M., and Masuda, S., "Characteristics of electric dust collector based on electric curtain", Proceedings of the General Conference of the Institute of Electronic Engineers in Japan, No. 821 (1971)

[7] Malnar, B., Balachandran, W., and Cecelja, F., "3D simulation of traveling wave dielectrophoretic force on particles", Proceedings of the ESA-IEEE Joint Meeting on Electrostatics 2003, Laplacian Press, Morgan Hill, CA, pp. $361-373$ (2003)

[8] Malyar, B., Kulon, J., and Balachandran, W., “Organization of particle sub-populations using dielectrophoretic force”, Proceedings of the ESA-IEEE Joint Meeting on Electrostatics 2003, Laplacian Press, Morgan Hill, CA, pp. 313-322 (2003)

[9] Masuda, S., "Electric Curtain for Confinement. and. Transport of Charged Aerosol Particles", Proc.of Albany Conference on Electrostatics, (1971)

[10] Jones, T.B., Electromechanics of Particles, Cambridge University Press, Cambridge (1995)

[11] Moesner, F.M. and Higushi, T., "Devices for particle handling by an AC electric field," Proceedings of IEEE Micro Electro Mechanical Systems, 66 pp. 66-71 (1995) 
[12] Zhou, G. Imamura, M., Suehiro, J., and Hara, M., "Enrichment and Recovery of Biological Cells Using a Dielectrophoretic Filter", Proceedings of the ESA-IEEE Joint Meeting on Electrostatics 2003, Laplacian Press, Morgan Hill, CA, pp. 337-348 (2003)

[13] Sims, R.A., Biris, A.S., Wilson, J.D., Yurteri, C.U., Mazumder, M.K., Calle, C.I., and Buhler, C.R., "Development of a Transparent dust shield for Solar Panels" Proc.of the ESA-IEEE Joint Meeting on Electrostatics 2003, Laplacian Press, Morgan Hill, CA, 814 (2003)

[14] Calle, C.I., A. Chen, J. Meyer, B. Linell, C.R. Buhler, S. Clements, and M.K. Mazumder, "Numerical modeling of an electrostatic dust shield for the Martian and lunar environments," Earth and Space 2006, Houston (2006)

[15] Calle, C.I., C. Immer, J.S. Clements, C. Buhler, A. Chen, J. Mantovani, P. Lundeen, and M. Michalenko, "Electrodynamic dust shield for surface exploration activities on the moon and Mars," Proceedings of the International Astronautical Congress, Valencia, Spain (2006)

[16] Mantovani, J.G. and C.I. Calle, "Controllable transport of particulate materials for in situ characterization," Proceedings of the 2007 IEEE Aerospace Conference, Big Sky, Montana (2007)

[17] Calle, C.I., J.S. Clements, C.R. Buhler, J.G. Mantovani, A. Chen, E.E. Arens, J.M. McFall, and M.L. Ritz, "Mitigation of Lunar Dust in Mechanical Systems," Proceedings of the Space Technologies and Applications International Forum, Albuquerque (2008).

[18] Calle, C.I., C.R. Buhler, J.G. Mantovani, S. Clements, A. Chen, M.K. Mazumder, A.S. Biris, and A.W. Nowicki, "Electrodynamic dust shield for Solar Panels on Mars, Lunar and Planetary Science XXXV, 2014 (2004)

[19] Atten, P., H.L. Pang, and J. Reboud, "Study of dust removal by standing wave electric curtain for application to solar cells on Mars," IEEE/IAS Annual Meeting, Hong Kong (2005)

[20] Kawamoto, H., "Some techniques on electrostatic separation of particle size utilizing electrostatic traveling wave," Journal of Electrostatics, 66, pp. 220-228 (2008)

[21] McKay, D.S., J.L. Carter, W.W. Boles, C.C. Allen, and J.H. Alton, "JSC-1: A new lunar regolith simulant," LPS XXIV, 963-964 (1993) 